\title{
STUDY OF BINDING INTERACTIONS OF HUMAN CARBONIC ANHYDRASE XII
}

\author{
HARSH BARUA, NIDHI BHAGAT, (MRS.) M. P. TORASKAR*
}

*Department of Pharmaceutical Chemistry, Bharati Vidyapeeth's College of Pharmacy, Navi Mumbai, India Email: mrunmayeetoraskar@gmail.com

Received: 02 Oct 2016, Revised and Accepted: 04 Dec 2016

\section{ABSTRACT}

Objective: The present study was carried out to study the binding interactions of different $\mathrm{N}^{\prime}$-(substituted phenyl sulfonyl)-pyridine-2carbohydrazide derivatives and N'-(substituted phenyl sulfonyl)-thiophene-2-carbohydrazide derivatives which were synthesized by senior students from research laboratory, with objective to explore the suitability of selected ligands for their binding affinity for the selected target.

Methods: Binding interactions of the selected ligands were studied using glide module of Schrodinger software using Maestro 10.1 interface. At the end of molecular docking studies, docking scores along with 2D and 3D binding interactions of these ligands were studied to evaluate the potency of ligands to act as selective human carbonic anhydrase (hCAXII) inhibitors in comparison with standard inhibitor Acetazolamide (AZA).

Results: Docking study on the ligands exhibited very similar conformation and binding interactions with hCAXII as that of standard. This suggests that selected ligands might possess significant binding affinity for hCAXII.

Conclusion: It can be concluded that the selected ligands have the potential to act as inhibitors of hCAXII.

Keywords: Molecular docking, Glide module, hCAXII, Acetazolamide

(C) 2016 The Authors. Published by Innovare Academic Sciences Pvt Ltd. This is an open access article under the CC BY license (http://creativecommons.org/licenses/by/4.0/] DOI: http://dx.doi.org/10.22159/ijcpr.2017v9i1.16633.

\section{INTRODUCTION}

The carbonic anhydrases (CAs, EC 4.2.1.1) are a family of enzymes that catalyze the rapid inter-conversion of carbon dioxide and water to bicarbonate and protons (or vice versa). The enzymes belong to class of metallo-enzymes as most of these enzymes have zinc ion in their active site. [1] They are present in both prokaryotes and eukaryotes, being encoded by four distinct, evolutionarily unrelated gene families: the $\alpha$-CAs (present in vertebrates, bacteria, algae and chloroplast of green plants), the $\beta$-CAs (predominantly found in bacteria, algae), the $\gamma$-CAs (mainly present in Archae and some bacteria) and the $\delta$-CAs (present in some marine diatoms). In mammals, 16 different $\alpha$-CA isoenzymes are divided into four subgroups, which, in turn consist of isoforms: cytosolic CAs (CA-I, CA-II, CAIII, CA-VII and CAXIII), mitochondrial CAs (CA-VA and CAVB), secreted CAs (CAVI) and membrane CAs (CA-IV, CA-IX, CA-XII, CA-XIV and CA-XV). These enzymes catalyze a very simple physiological reaction, the interconversion between carbon dioxide and the bicarbonate ion, and are thus involved in crucial physiological processes connected with respiration and transport of $\mathrm{CO}_{2}$ /bicarbonate between metabolizing tissues and lungs, $\mathrm{pH}$ and $\mathrm{CO}_{2}$ homeostasis, electrolyte secretion in a variety of tissue/organs, biosynthetic reactions (such as gluconeogenesis, lipogenesis and ureagenesis), bone resorption, calcification, tumorigenicity and many other physiologic or pathologic processes.

$$
\mathrm{H}_{2} \mathrm{O}+\mathrm{CO}_{2} \stackrel{\mathrm{CA}}{\longleftrightarrow} \mathrm{H}^{+}+\mathrm{HCO}_{3}^{-}
$$

\section{Mechanism}

The Zn (II) ion of CAs is essential for catalysis. X-ray crystallographic data showed that the metal ion is coordinated by three histamine residues (His 94, His 96, and His 119) and a water molecule/ hydroxide ion. The zinc bound water is also engaged in hydrogen bond interactions with the hydroxyl moiety of Thr 199, which in turn is bridged to the carboxylate moiety of Glu 106; these interactions enhance the nucleophilicity of the zinc-bound water molecule, and orient the substrate $\left(\mathrm{CO}_{2}\right)$ in a favorable location for the nucleophillic attack.

1. The active form of the enzyme is the basic one, with hydroxide bound to $\mathrm{Zn}$ (II) [A]. This strong nucleophile attacks the $\mathrm{CO}_{2}$ molecule bound in a hydrophobic pocket in its neighborhood [B], leading to formation of bicarbonate coordinated to $\mathrm{Zn}$ (II) [C]

2. The bicarbonate ion is then displaced by a water molecule and liberated into solution [D], leading to the acid form of the enzyme, with water coordinated to $\mathrm{Zn}$ (II), which is catalytically inactive.

3. In order to regenerate the basic form, a proton transfer reaction from the active site to environment takes place, which may be assisted by active site residues or by buffers in the medium.

4. The rate limiting step of the reaction is the second step in which hydroxide is regenerated by proton transfer. The reaction is schematically represented as follows:

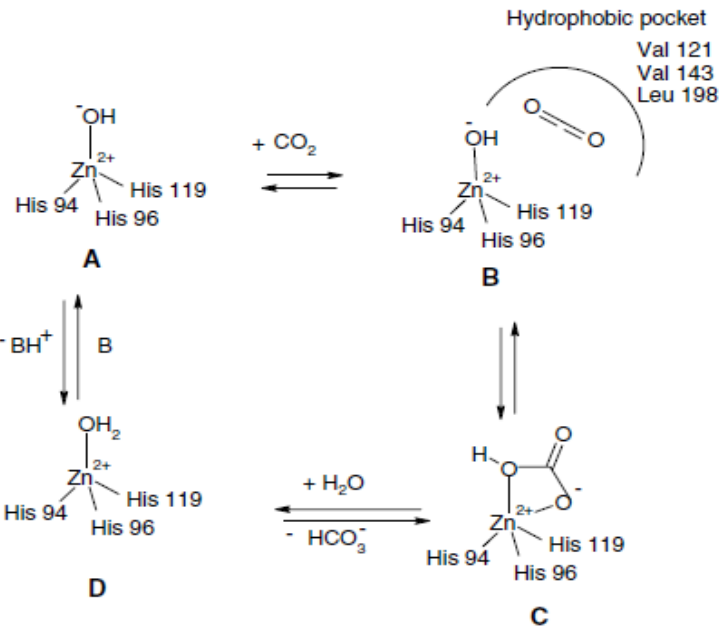

Fig. 1: Schematic representation of general mechanism of action of CA

Human carbonic anhydrase XII

CAXII is expressed in a variety of normal human tissues including kidney, colon, prostrate, pancreas, ovary, testis, lung and brain. 
Expression of hCAXII usually becomes stronger or more widespread in tumors compared to the corresponding normal tissues. This characteristic of CAXII can act as potential target for anticancer studies. In addition to anticancer studies it can also act as potential target for treating various diseased conditions like glaucoma, hypertension and epilepsy [2]. The isozyme has low catalytic activity with high affinity for sulfonamide.

\section{Carbonic anhydrase inhibitors}

Two main classes of CA inhibitors are known: the metal complexing anions and the un-substituted sulfonamides, which bind to $\mathrm{Zn}$ (II) ion of the enzyme either by substituting the nonprotein zinc ligand or add to the metal coordination sphere, generating trigonal-bipyrimidal species. Sulfonamides, which are the most important CAIs bind in tetrahedral geometry of the $\mathrm{Zn}$ (II) ion, in deprotonated state, with the nitrogen atom of the sulfonamide moiety coordinated to $\mathrm{Zn}$ (II) and an extended network of hydrogen bonds, involving residues Thr199 and Glu 106 , also participating to the anchoring of the inhibitor molecule to the metal ion. The aromatic/heterocyclic part of the inhibitor interacts with hydrophilic and hydrophobic residues of the cavity $[3,4]$. Anions may bind either in tetrahedral geometry of the metal ion or as trigonal-bipyramidal adducts, such as for instance the thiocyanate adduct as shown in fig.

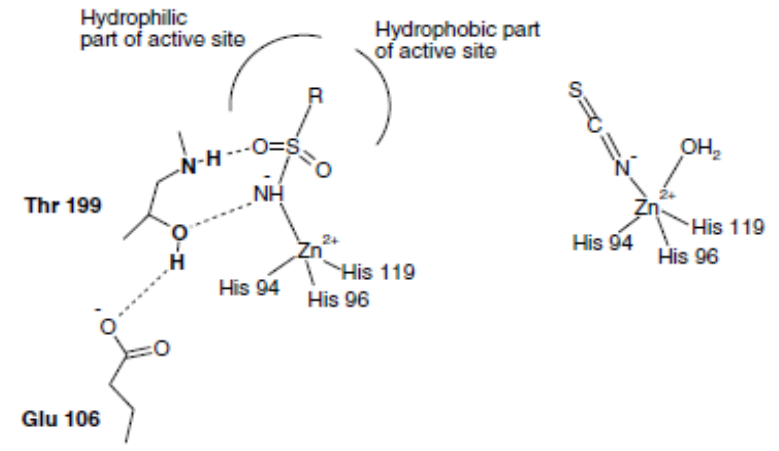
Tetrahedral adduct
(sulfonamide)

\section{A}

Fig. 2: Inhibition mechanism by sulfonamide (A) and anionic (B) inhibitors

\section{Molecular docking}

Molecular docking is a widely-used computational tool for the study of molecular recognition, which aims to predict the binding mode and binding affinity of a complex formed by two or more constituent molecules with known structures. An important type of molecular docking is protein-ligand docking because of its therapeutic applications in modern structure-based drug design. Structure (target)-based drug design represents docking i.e. ligand binding to its receptor, target protein. Docking is used to identify and optimize drug candidates by examining and modeling molecular interactions between ligands and target macromolecules. In the process of docking, multiple ligand conformations and orientations are generated and the most appropriate ones are selected. Scoring functions are fast approximate mathematical methods used to predict the strength of the non-covalent interaction (also referred to as binding affinity) between two molecules after they have been docked. General observation is that consensus (combination of different scoring algorithms) scoring yields better results than individual scoring. Validations may be performed with known active and inactive ligands, comparisons to crystallographic data and prediction of rank-ordering and binding affinities.

To perform this molecular docking we have used glide module of Schrodinger software using Maestro10.1 interface.

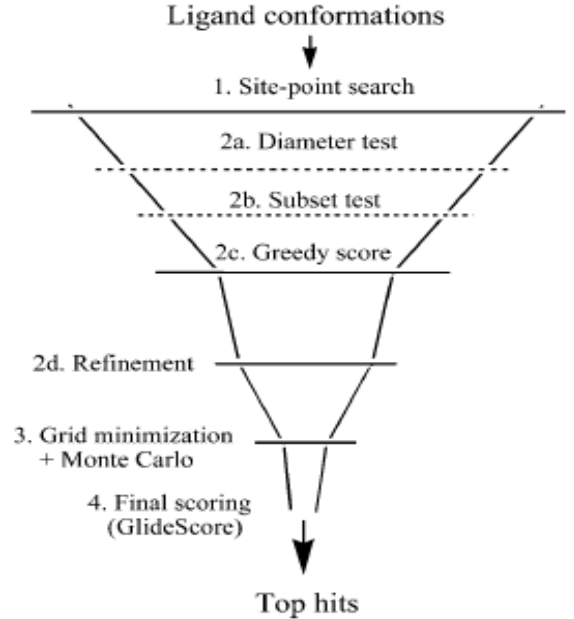

Fig. 3: Glide docking funnel, showing the glide docking hierarchy

\section{Rationale}

Human Carbonic Anhydrase XII (hCA XII) is a membrane-tethered enzyme that is highly expressed in many human tumors. The catalytic activity of carbonic anhydrases has been demonstrated to also facilitate tumor metabolism and thus to trigger tumor growth Inhibition of hCAXII can be used as a novel means of targeting cancer. Many of the available drugs in the market have nonselective inhibition for the different isoforms of Carbonic Anhydrase. Thus there is critical need for the development of drugs that would selectively target tumor associated isoform hCAXII. Here in our report we use the ligands designed by seniors in research laboratory which have sulfonamide group in common to carry out docking studies on our target hCAXII, a potential clinically useful tumor marker. To determine the potency of these ligands as hCAXII inhibitors we use acetazolamide as a standard for comparison.

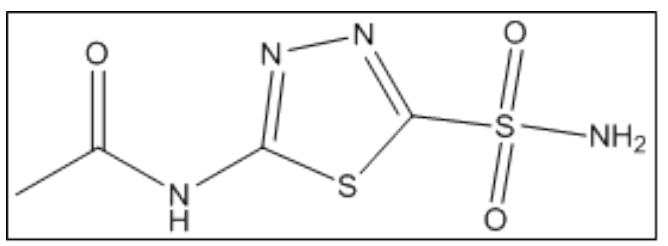

Fig. 4: Structure of standard Acetazolamide

We evaluated these ligands on the basis of their docking score which reflects the binding affinity for the target. These docking scores are determined by use of glide module of Schrodinger software using Maestro interface 10.1. These ligands if found potent enough would lead to development of novel therapeutic anticancer agents which would have advantages of selective in action and lesser side effects as compared to existing cancer chemotherapy. The aim of this study is to identify the various binding interactions of the selected ligands for hCAXII and thus evaluate its binding affinity for the enzyme.

\section{MATERIALS AND METHODS}

\section{Selection of ligand}

From the outcome of the literature survey, it was observed that sulfonamide functional group is essential for the binding to the active site of carbonic anhydrase which finally leads to its inhibition. Taking into consideration pharmacophore requirements of carbonic anhydrase, we have used the ligands designed by seniors which have sulfonamide group to carry out docking studies. These ligands are $\mathrm{N}$-(substituted phenyl sulfonyl)-thiophene-2-carbohydrazide derivatives and $\mathrm{N}^{\prime}$ (substituted phenyl sulfonyl)-pyridine-2-carbohydrazide derivatives. 


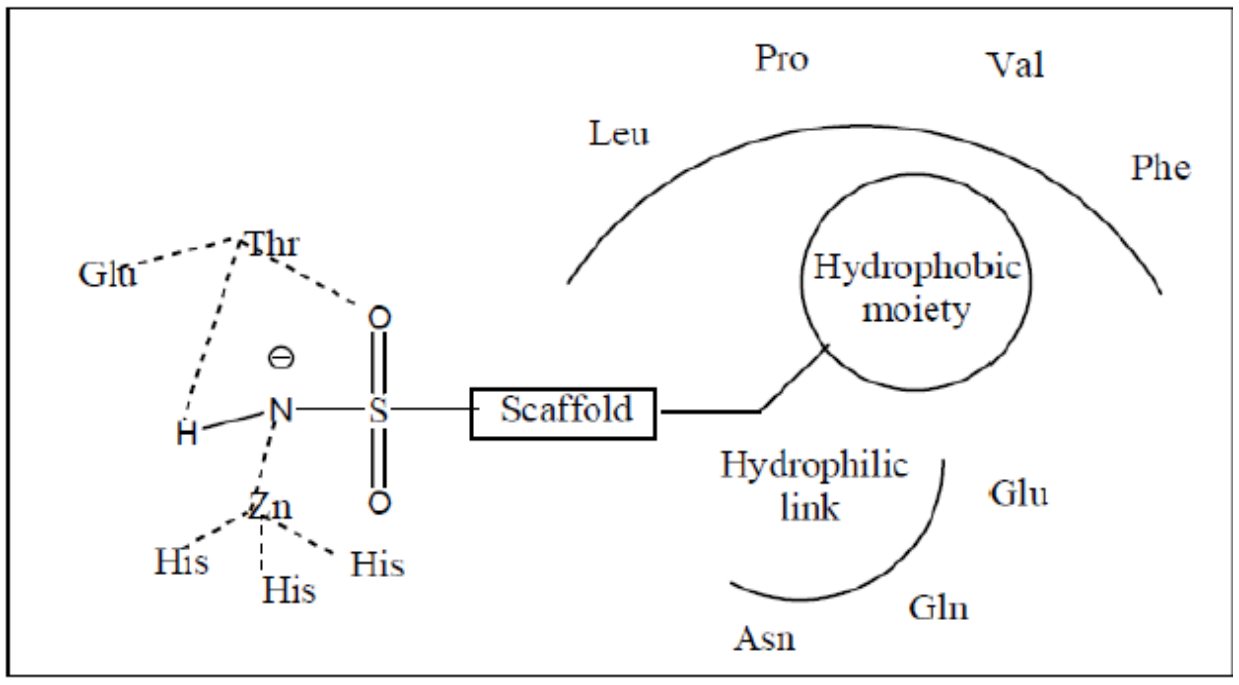

Fig. 5: Pharmacophore requirement of CA inhibitors in the CA enzymatic active site

The structures of ligands used for the studies with their assigned code are as follows:

Table 1: Structures of different substituents along with their assigned codes

I) $\quad \mathrm{N}^{\prime}$-(substituted phenyl sulfonyl)-thiophene-2-carbohydrazide derivatives<smiles>O=C(NNS(=O)(=O)[Al])c1cccs1</smiles>

N'-(substituted aryl sulfonyl)-thiophene-2-carbohydrazide

Code


<smiles>O=C(NNS(=O)(=O)[AlH2])c1ccccn1</smiles>

N'-(sustituted aryl sulfonyl)-pyridine-2-carbohydrazide

4-methyl phenyl

\section{Molecular Docking studies}

Molecular docking studies are used to determine the strength of the interactions and to find out the best orientation of ligand which would form a complex with protein with minimum energy. Docking studies were performed using Maestro 10.1 interface of Schrodinger software using glide module, this included following steps.

Protein Preparation: This process was initiated by importing protein structure from protein data bank. Our protein Carbonic Anhydrase XII had the PDB ID 1JD0 which was used to download the protein structure. The unprepared protein was then subjected to series of modification under command PrepWiz which was used to preprocess, delete the $\mathrm{B}$ chain of protein, further all the water molecules that were in coordination with A chain were also deleted. In the end metal binding states were generated and ultimately protein structure was subjected to optimization and minimization. After going through all these steps final protein was ready for docking.

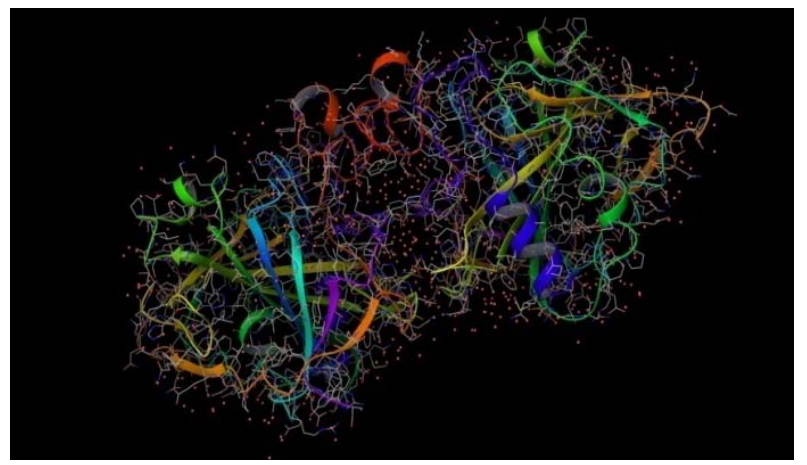

Fig. 6: 3D structure of CAXII that was imported from PDB using ID 1JD0

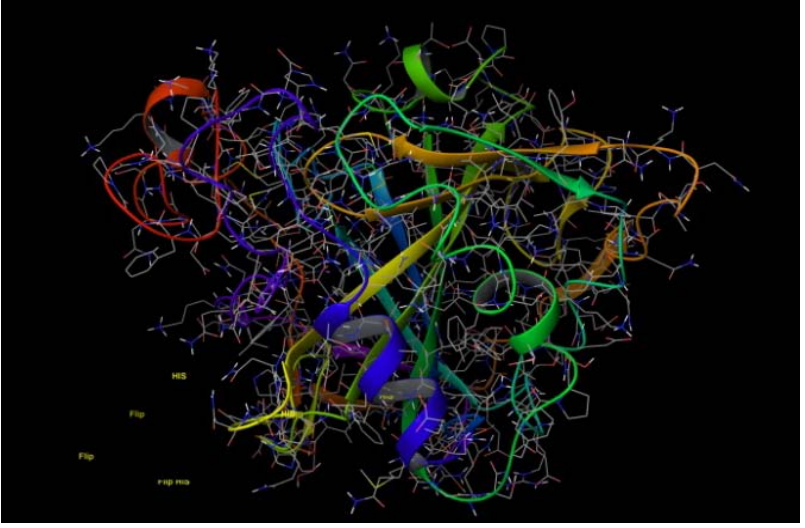

Fig. 7: 3D structure of CAXII after protein preparation

Ramachandran Plot: The Ramachandran Plot panel displays a plot of the dihedral angles $\varphi$ and $\psi$ for each residue in the protein that is displayed in the Workspace. The plot area displays a plot of protein dihedrals for all residues in the protein. The darkest area "red region" corresponds to "core" region representing the most favorable combinations. Ideally $90 \%$ of the residues should be in this "core" region.

In this way we evaluated structure of our prepared protein which had the maximum residues in the core region. This indicated that the modifications done had not disturbed the protein structure.

Receptor Grid Generation: It is done to search for the favorable interactions between ligand molecules and a receptor molecule. The receptor grid was set up and generated from the Receptor Grid Generation panel. 


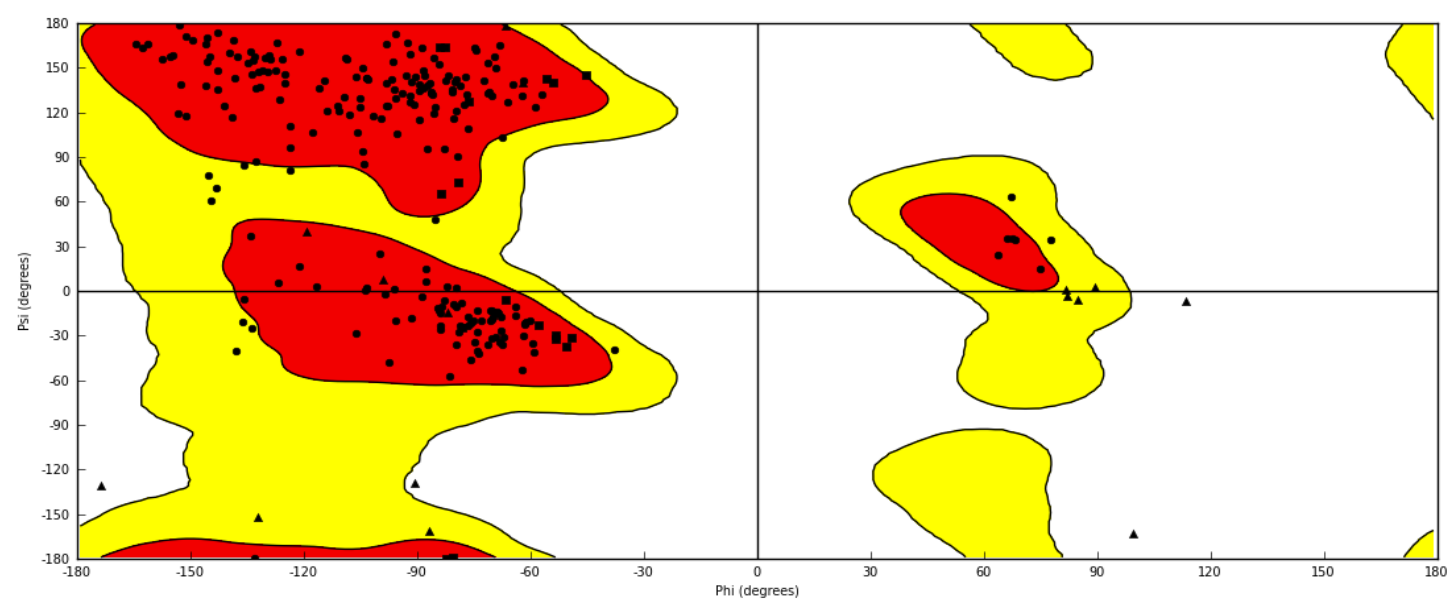

Fig. 8: Ramachandran plot of the prepared protein 1JD0

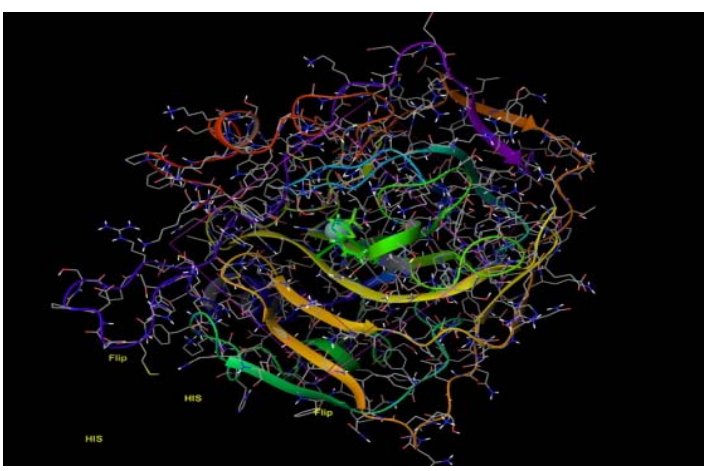

Fig. 9: 3D structure of CAXII after grid generation

Ligand Preparation: After the preparation of the protein, ligands prepared to dock in the active site of protein was subjected to the LigPrep process which consists of a series of steps that perform conversions, corrections to the structures, generating variations on the structures, eliminating unwanted structures, and optimising the structures. The preparation involves the following tasks:

1. The addition of hydrogen atoms.

2. Filtering unsuitable molecules based on their properties.

3. Removing any unwanted molecules, such as water, small ions.

4. Neutralizing charged groups, then the generation of ionisation and tautomeric state.
5. Generation of stereoisomers.

6. Generation of low-energy ring conformations.

7. Removing of any badly prepared structures.

8. Optimising the geometries.

In this way, ligand preparation was completed.

Ligand Docking: Once protein and ligand preparation is completed, prepared ligands were studied for their affinity for the protein by use of glide docking. We have studied the binding interactions between the protein and all possible conformations of the ligands. In the Glide docking module, Glide generates a set of conformers for each ligand and then performs an exhaustive search for possible positions and orientations of ligand over the active site. These conformers pass through a series of hierarchical filters (docking score, docking pose, docking energy, hydrogen bonding, etc) that evaluate the interaction of the ligand with the receptor. Poses are then subjected to energy minimization on pre-computed van der Waals and electrostatic grids of the receptor. Final scoring is then carried out on the energy-minimized poses. At the end docking score for each conformer for the ligand is obtained from the project table which is noted.

\section{RESULTS}

\section{Project table}

Docking scores of the ligands that were obtained at the end of docking were tabulated. Molecular docking of standard acetazolamide was carried using glide docking and corresponding docking score which was obtained, was set as a standard.

Table 2: Docking scores of the ligands docked on hCAXII isozyme

\begin{tabular}{llc}
\hline Code & IUPAC & Docking score \\
\hline Standard & & (Acetazolamide) \\
\multicolumn{2}{c}{ Thiophene Derivatives } & -6.711 \\
01 & N'-(thiophene-2-carbonyl)benzenesulfonohydrazide & -4.576 \\
02 & 4-chloro-N'-(thiophene-2-carbonyl)benzenesulfonohydrazide & -5.351 \\
03 & 4-nitro-N'-(thiophene-2-carbonyl)benzenesulfonohydrazide & -5.149 \\
04 & 4-fluoro-N'-(thiophene-2-carbonyl)benzenesulfonohydrazide & -4.877 \\
05 & 4-methyl-N'-(thiophene-2-carbonyl)benzenesulfonohydrazide & -4.246 \\
06 & 4-chloro-3-nitro-N'-(thiophene-2-carbonyl)benzenesulfonohydrazide & -5.333 \\
07 & 4-methoxy-N'-(thiophene-2-carbonyl)benzenesulfonohydrazide & -4.065 \\
Pyridine Derivatives & -5.222 \\
08 & 4-chloro-N'-picolinoylbenzenesulfonohydrazide & -4.886 \\
09 & 4-nitro-N'-picolinoylbenzenesulfonohydrazide & -4.795 \\
10 & 4-fluoro-N'-picolinoylbenzenesulfonohydrazide & -4.262 \\
11 & 4-methyl-N'-picolinoylbenzenesulfonohydrazide & -5.011 \\
12 & 4-chloro-3-nitro-N'-picolinoylbenzenesulfonohydrazide & -5.424 \\
13 & 4-methoxy-N'-picolinoylbenzenesulfonohydrazide & \\
\hline
\end{tabular}


Molecular Docking of N-(5-sulfamoyl-1,3,4-thiadiazol-2-yl) acetamide:
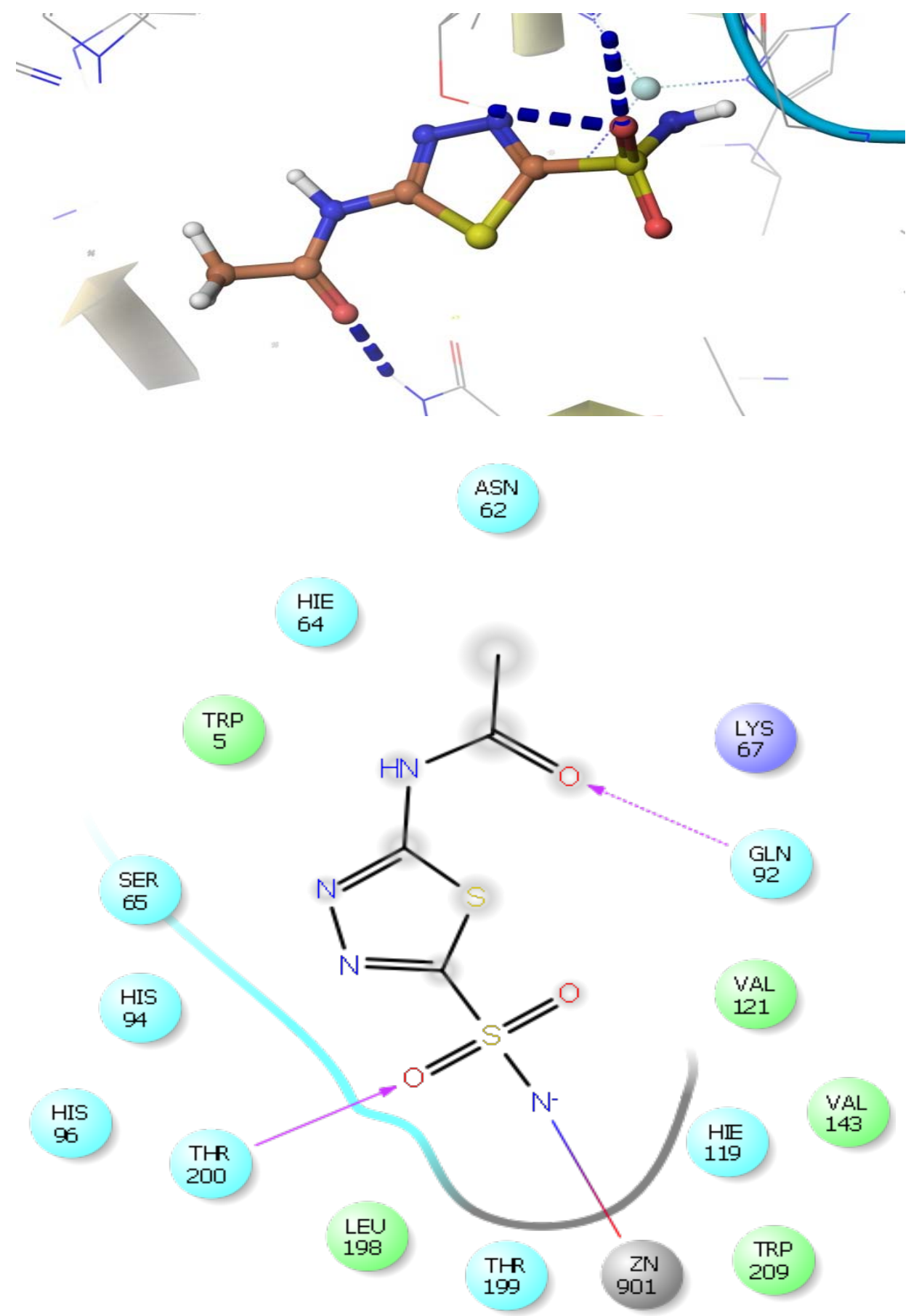

Fig. 10: 3D ligand-protein binding interaction of AZA at CA XII binding site

$\begin{array}{llll}\text { Charged (negative) } & \text { Polar } & \cdots \text { Distance } & \text { Salt bridge } \\ \text { Charged (positive) } & \text { Unspecified residue } & \rightarrow \text { H-bond (backbone) } & \text { Solvent exposure } \\ \text { Glycine } & \text { Water } & \cdots \text { H-bond (sidechain) } & \\ \text { Hydrophobic } & \text { Hydration site } & - \text { Metal coordination } \\ \text { Metal } & \text { Hydration site (displaced) } & \rightarrow \text { Pi-Pi stacking }\end{array}$

Fig. 11: 2D ligand-protein binding interaction of AZA at CA XII binding site

Docking of the standard compound showed the presence of very strong binding affinity for the active site of CAXII. The nitrogen of sulfonamide functional group is in coordination with the zinc ion of the active site. Further, the interactions of the AZA are strengthened by hydrogen bonding with surrounding amino acid residues. 
Molecular docking of best scored confirmation of ligands

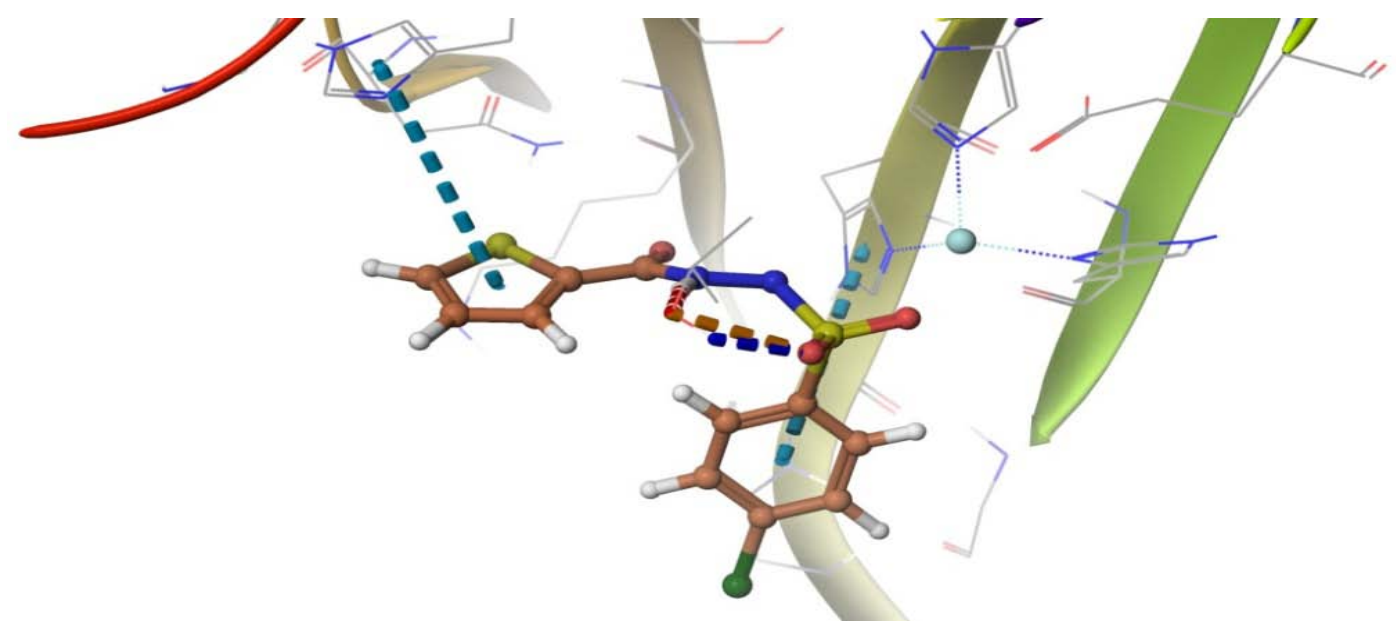

Molecular Docking showing binding of 4-chloro-N'-(thiophene-2-carbonyl) benzenesulfonamide (Code: 02)

II) Fig. 12: 3D ligand-protein binding interaction of ligand code 02at CA XII binding site

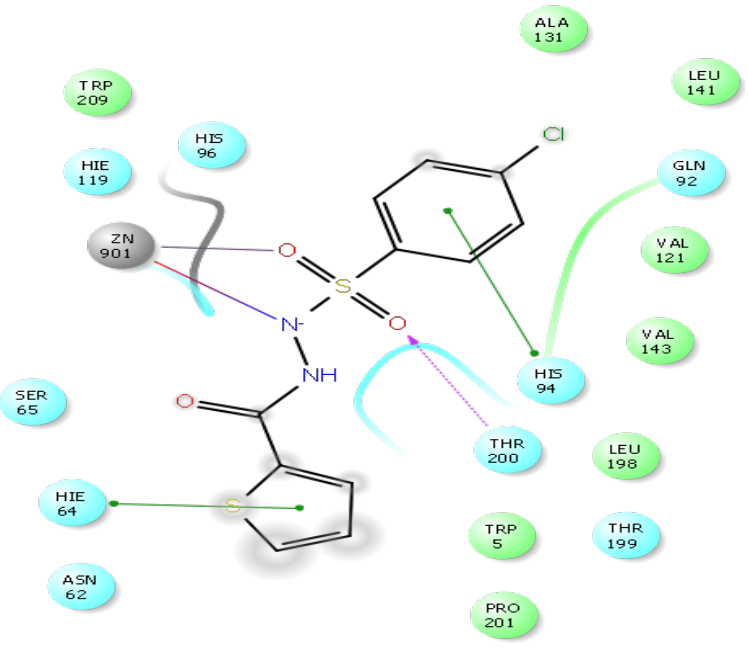

Fig. 12: 2D ligand-protein binding interaction of ligand code 02 at CA XII binding site

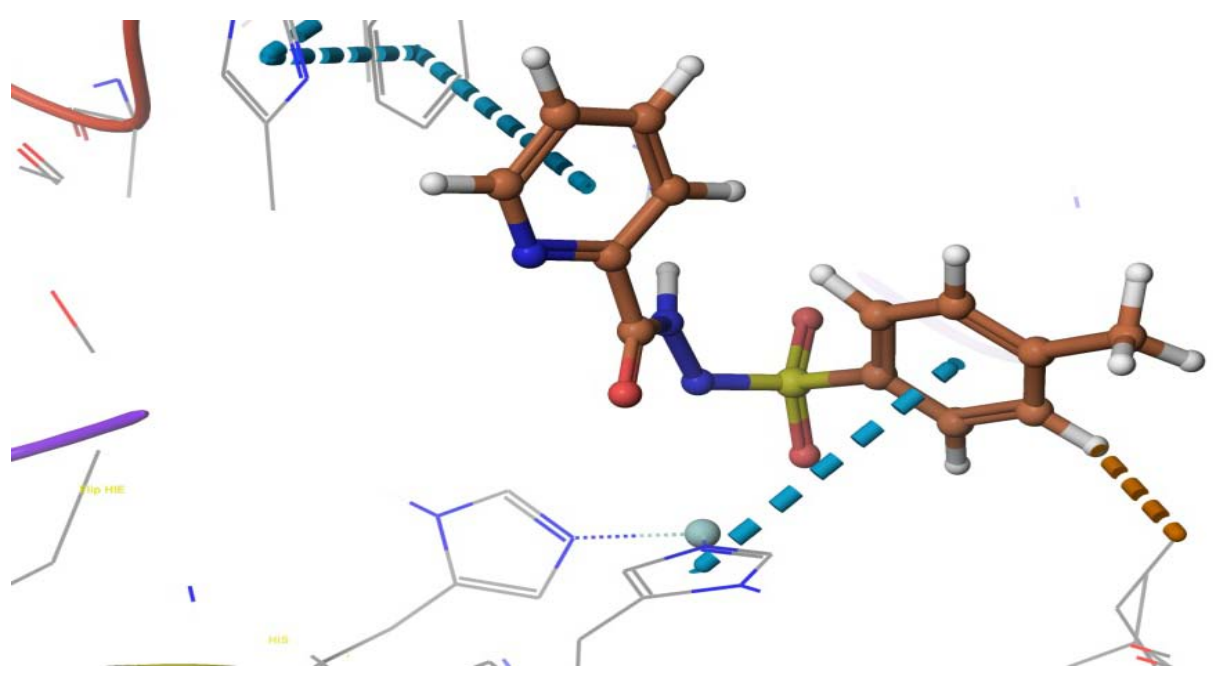

Fig. 13: 3D ligand-protein binding interaction of ligand code 13 at CA XII binding site 


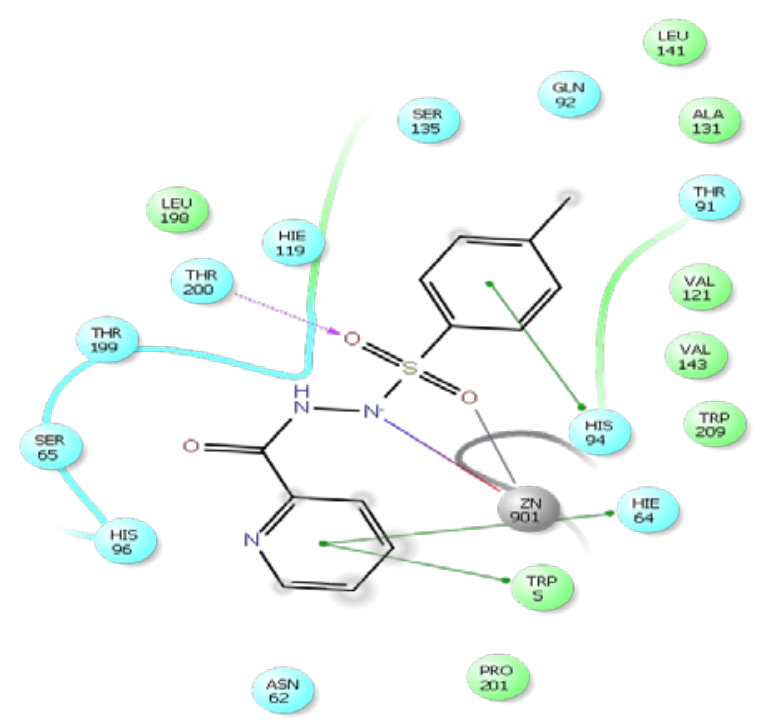

Fig. 14: 2D ligand-protein binding interaction of ligand code 13 at CA XII binding site

\section{DISCUSSION}

The docking scores along with 2D and 3D binding interactions of the best-scored ligand from each group of derivatives were carefully studied. This showed us that the sulfonamide group of the ligand was found to chelate with the zinc ion of the active site of target same as that of the standard. These interactions are essential for the ligands to show binding to hCAXII. In addition to the chelation with the zinc atom, ligands were found to interact with amino acid residues of the hCAXII in the form of hydrogen bonding and pi-pi stacking. Hydrogen bonding occurs due to the hydrogen bond acceptor oxygen that is present in the sulphonamide moiety. Further pi-pi stacking are the attractive, noncovalent interactions and these occur due to interactions between aromatic ring of ligand and protein. These interactions of the ligand with hCAXII observed in best-docked poses were similar to that observed in the standard acetazolamide. This enhances the possibility if ligands are to be used as hCAXII inhibitors in the near future. The docking score of both ligand and standard are shown in the table above. Further docking score of other docked ligands are also included in the table for the comparison. The ligand code 02 from thiophene derivatives and ligand code 13 from pyridine derivatives were found to show the highest docking score from their respective groups. These scores were good, as they were near to standard acetazolamide docking score. Finally, it is clear that small modification in the scaffold of ligand leads to differences in the docking scores when docked on target hCAXII. Therefore, it can be said that docking study on designed ligands exhibited very similar conformation and binding interactions with hCAXII as that of the standard. This suggests that selected ligands might possess a significant binding affinity for hCAXII.

\section{CONCLUSION}

Designing the ligands that would selectively inhibit tumor-associated hCAXII isoform over the other isoforms was a critical issue as mentioned in our rationale of the report. In our report, we have carried out docking studies for the ligands that have a potential selective affinity for hCAXII. In summary, 13 ligands were docked on target hCAXII and all had similar docking score as that of the standard. Finally, we report a series of N'-(substituted phenyl sulfonyl)-pyridine2-carbohydrazide derivatives and $\mathrm{N}^{\prime}$-(substituted phenyl sulfonyl)thiophene-2-carbohydrazide derivatives that were evaluated in docking studies for their binding affinity for target hCAXII. These derivatives were found to have an affinity for the target hCAXII. Further studies should be done to modify, explore in detailed mechanism of action and to broaden the structure-activity relationship for this interesting class of sulfonamide hCAXII inhibitors.

\section{ACKNOWLEDGEMENT}

Authors are thankful to Dr. Vilasrao J. Kadam, Principal, Bharati Vidypeeth's College of Pharmacy, Navi Mumbai, for providing all the required facilities like library, computer laboratory, the internet and all that was required to carry out our research work successfully. We would also like to thank Pravin and Santoshi from S. Y. M. Pharm for helping us during the entire duration of our research work.

\section{CONFLICT OF INTERESTS}

\section{Declared none}

\section{REFERENCES}

1. Supuran CT, Scozzafava A. Carbonic anhydrases as targets for medicinal chemistry. Bioorg Med Chem Lett 2007;15:4336-50.

2. Vullo D, Innocenti A, Nishimori I, Pastorek Jaromí r, Scozzafava A, Pastoreková, et al. Carbonic anhydrase inhibitors. inhibition of the transmembrane isozyme XII with sulfonamides-a new target for the design of antitumor and antiglaucoma Drugs? Bioorg Med Chem Lett 2005;15:963-9.

3. Durdagi S, Sentürk M, Ekinci D, Balaydın HT, Göksu S, Küfrevioğlu ÖI, et al. Kinetic and docking studies of phenolbased inhibitors of carbonic anhydrase isoforms I, II, IX and XII evidence a new binding mode within the enzyme active site. Bioorg Med Chem Lett 2011;19:1381-9.

4. Nordfors K, Haapasalo J, Korja M, Niemelä A, Laine J, Parkkila $\mathrm{AK}$, et al. The tumour-associated carbonic anhydrases CA II, CA IX and CA XII in a group of medulloblastomas and supratentorial primitive neuroectodermal tumours: an association of CA IX with poor prognosis. BMC Cancer 2010;10:148.

5. Alafeefy AM, Isik S, Abdel-Aziz HA, Ashour AE, Vullo D, Al-Jaber NA, Supuran CT. Carbonic anhydrase inhibitors: benzenesulfonamides incorporating cyanoacrylamide moieties are low nanomolar/subnanomolar inhibitors of the tumorassociated isoforms IX and XII. Bioorg Med Chem Lett 2013;21:1396-403.

6. Güzel-Akdemir Ö, Akdemir A, Isik S, Vullo D, Supuran CT. oBenzenedisulfonimido-sulfonamides are potent inhibitors of the tumor-associated carbonic anhydrase isoforms CA IX and CA XII. Bioorg Med Chem Lett 2013;21:1386-91.

\section{How to cite this article}

- Harsh Barua, Nidhi Bhagat, (MRS.) MP Toraskar. Study of binding interactions of human carbonic anhydrase XII. Int J Curr Pharm Res 2017;9(1):118-125. 